How to reference this article Lenart, M. (2021). Un messaggio per i posteri. La tomba di Stanisław Miński nella Basilica di Sant'Antonio a Padova. Italica Wratislaviensia, 12(2), 11-27.

DOI: http://dx.doi.org/10.15804/IW.2021.12.2.01

\author{
Mirosław Lenart \\ Uniwersytet Opolski, Polonia \\ lenart@uni.opole.pl \\ ORCID: 0000-0001-9184-6893
}

\title{
UN MESSAGGIO PER I POSTERI. LA TOMBA DI STANISLAW MIŃSKI NELLA BASILICA DI SANT'ANTONIO A PADOVA
}

\author{
A MESSAGE TO POSTERITY: \\ THE TOMB OF STANISŁAW MIŃSKI \\ IN THE BASILICA OF ST. ANTHONY IN PADUA
}

\begin{abstract}
The article deals with the first burial in the tomb crypt built under the altar of the natio polona in the Basilica of St. Anthony in Padua. Stanisław Miński, a Polish state dignitary and diplomat who was passing through Padua on his way from Naples, was the one buried here. The tombstone, removed only in the first half of the $20^{\text {th }}$ century, contained information about the laying of Miński's body to rest in the place where church services associated with Poland and Poles were performed. For over three centuries, the tomb attracted the attention of pilgrims from Poland coming to Padua, reminding them not only of the outstanding Polish diplomat, but also of the natio polona, which, in the $16^{\text {th }}$ and early- $17^{\text {th }}$ centuries, constituted a large and distinctive group of ultramontani, that is, those who came from beyond the mountains, most of whom had come to study at the flourishing university. In the article, little-known documents concerning the burial are gathered; these documents are kept in the Paduan archives and had been collected by the clergy and secular administration of the Basilica. Via the analysis of these documents, the cultural context in which the decision against bringing Miński's body to Poland is reconstructed. The role of the institution, constituted by the administration of St. Anthony's Basilica operating under the name of Veneranda Arca di Sant'Antonio, which grants permission to lay the body in the Basilica, is also established.
\end{abstract}

Keywords: Stanisław Miński, Padua, natio polona, Basilica of St. Anthony, tomb 
Drima di ripercorrere più da vicino le vicende legate alla sepoltura 1 di Stanisław Miński (1561 ca. -1607), voivoda di Łęczyca, vicecancelliere della Corona del Regno di Polonia, consigliere fidato di Sigismondo III e messo diplomatico (Gruszecki, 1976, pp. 321-322), dobbiamo soffermarci sul luogo in cui furono deposte le spoglie mortali del diplomatico polacco, indicato da una lapide con epitaffio sulla quale ci sarà ancora occasione di ritornare. Si tratta della Basilica di Sant'Antonio a Padova, la quale costituisce uno spazio importante, non solo in considerazione del culto di uno dei patroni della città, bensì anche dell'altare eretto per volontà della natio polona nel 1607. Se non si menzionasse questo centro della vita religiosa dei polacchi che professavano il cattolicesimo, contraddistinti dallo zelo tipico dell'epoca postridentina, il racconto della commemorazione di Stanisław Miński sarebbe incompleto. La storia dell'erezione dell'altare è stata di recente trattata dettagliatamente, come del resto la sepoltura stessa del sottocancelliere della Corona del Regno di Polonia, in studi separati, che costituiscono il frutto di un progetto di ricerca da me condotto (Lenart, 2019b, pp. 145-187; Wrana, 2019, pp. 214-215, 259-260). Negli studi suddetti, principalmente di carattere documentalistico, mancava tuttavia lo spazio per analizzare più da vicino i testi stessi, sia quelli incisi nella pietra, che per oltre tre secoli attirarono l'attenzione dei peregrinanti giunti a Padova al cospetto del santuario, innalzato in onore del Grande Taumaturgo, sia quelli conservati fra le mura dei locali archivi. Ricordiamo che l'altare sopraccitato fu eretto sopra alla cripta principale, in cui il corpo di Miński aveva trovato riposo per primo. Questo fatto rappresenta il punto di partenza delle riflessioni proposte in questa sede, poiché i monumenti funebri sono di per sé importanti testi culturali, inseriti nel dialogo condotto dall'uomo con il mondo che lo circonda.

La scelta della chiesa da parte dei rappresentanti della natio polo$n a$, di una chiesa che assumeva un'importanza sempre maggiore nella vita della città e dell'università, specie dopo il 1592 (Lenart, 2019a, pp. 143-164), era dovuta di certo alla sua contiguità rispetto al luogo in cui abitavano i polacchi. Ne sono una chiara testimonianza le esequie di Erazm Kretkowski, deceduto a Padova nel 1558, nonché il fatto di avergli eretto un monumento funebre (Lenart, 2010, pp. 64-75; Wrana, 
2019, pp. 192-203, 238-254). Si trattava con certezza del monumento più imponente legato alla Polonia, anche se va rilevato che nel 1585 sulla parete di uno dei chiostri del convento venne affissa la lapide con epitaffio di Andrzej Kański (Wrana, 2019, pp. 203-205). La questione della donazione della cripta funebre, destinata all'inumazione, fu discussa già in occasione della prima seduta della natio polona, riorganizzatasi, il 20 dicembre 1592 (Archivio della nazione polacca dell'Università di Padova, vol. 2, pp. 19-22; Del Negro, \& Piovan, 2017, pp. 513-518). La sua costruzione assieme all'altare iniziò tuttavia a prendere forma concreta solo nel 1606, il che era dovuto anche alla ricerca di una più degna sua collocazione (ASP, SA 2) ${ }^{1}$. Alla fine fu scelta la parte presso la porta, a sinistra della navata. Non era un sito particolarmente prestigioso, poiché un tempo attraverso la suddetta porta si lasciava la basilica, conformemente al percorso tracciato per coloro che visitavano il santuario, in cui la tomba di Sant'Antonio costituiva il punto di riferimento. Occorre tuttavia tenere presente che l'interno della basilica a quel tempo non abbondava di spazi vuoti, soprattutto per quanto concerne la possibilità di erigere una cripta funebre. Il luogo destinato alla costruzione dell'altare e della cripta, associato in seguito con la natio polona, era contraddistinto dal fatto che sulla parete si trovava una decorazione con stelle dipinte. Molto probabilmente si trattava di evocare il significato, un tempo ben leggibile, della volta celeste, di cui un esempio eloquente si trova nella Cappella degli Scrovegni di Padova, dove possiamo ammirare gli affreschi di Giotto. Nel Giudizio Universale là rappresentato vediamo gli angeli che arrotolano la volta del cielo, svelando l'architettura della Gerusalemme celeste. Questa rappresentazione ricordava come il mondo che ci circonda sia una specie di scenografia per quello spettacolo che è la storia dell'umanità, nel quale l'Incarnazione del Figlio di Dio costituisce il momento cruciale. Le riflessioni attorno alla comparsa terrena su questa scena di certo non erano estranee nemme-

1 Il documento riguarda l'assegnazione da parte dei Francescani dello spazio destinato alla cappella nazionale e alla sepoltura delle salme contenute nella cappella della famiglia Turchetto (successori dei Lanzarotti), ovvero alla cappella delle Stimmate. In questo caso, tuttavia, lo Spitale di San Francesco che la gestiva, si oppose alla decisione. 
no ai polacchi che operavano per far erigere la cripta e l'altare, come dimostra l'iscrizione di carattere moralistico-religioso che una volta si trovava sulla lapide posta a destra:

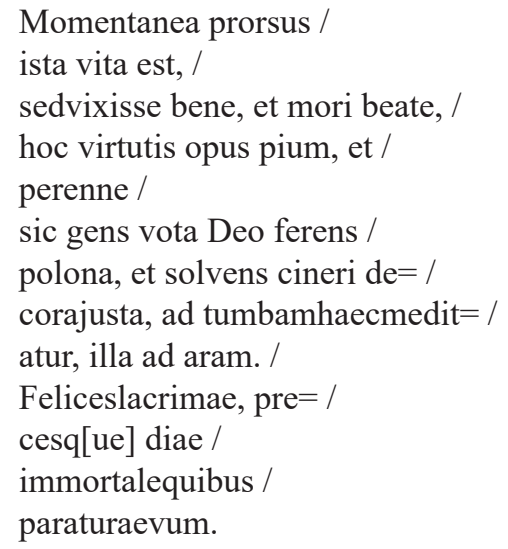

La vita è qualcosa che dura solo un attimo, ma vivere bene e morire felicemente è nobile e duratura opera di virtù - così la natio polona, implorante Dio con la preghiera e rendendo i giusti onori alle ceneri, sull'uno medita davanti alla tomba, sull'altro davanti all'altare. Lacrime felici e sante preghiere destinate a perdurare immortali nei secoli.

Nel testo qui riportato sono contenuti i valori fondamentali a cui si ispiravano in vita, e che intendevano trasmettere ai posteri, i rappresentanti della nobiltà polacca aventi aspirazioni umanistiche. Un concetto-chiave è qui la virtù la quale, in quanto valore costruito e duraturo, rappresenta non solo il decoro dell'uomo impegnato nel vivere la fede, bensì costituisce il segno distintivo del rappresentante di quello stato che si identifica con la natio polona. Il messaggio di questa frase latina era un tempo legato alla presenza dell'altare, che rappresentava, secondo Jerzy Kowalczyk, il tipo da lui chiamato "araldico": "nel dossale, come spesso avveniva da noi, c'era un gruppo di stemmi che simboleggiava in questo caso tutta la Repubblica" (Kowalczyk, 1967, p. 78). Il mausoleo polacco costituiva dunque fin dagli inizi un importante sfondo per le azioni liturgiche, il cui fine era non solo quello di rendere omaggio a Dio, ma anche di promuovere la cultura polacca, se non altro attra- 
verso la celebrazione degli anniversari delle battaglie vittoriose, in cui virtù quali la fede ed il coraggio costituivano riferimenti automatici alla nazione degli eroi posti a guardia del Cristianesimo, a difesa degli estesi confini che dividevano l'Europa da un mondo recepito come aggressivo e ostile nei confronti della religione avente come proprio centro Roma. Di perpetuare l'immagine della Polonia come antemurale del Cristianesimo si occupavano, tra gli altri, i diplomatici polacchi diretti nella Città Eterna, cosa di cui troviamo esempi significativi anche nella Basilica di Sant'Antonio a Padova (Lenart, 2019c, pp. 65-74). Ad essi apparteneva anche Stanisław Miński, a cui non fu dato di rientrare in patria. Tuttavia, sia ha l'impressione che la sua missione diplomatica non sia terminata con la sua scomparsa, poiché egli divenne una sorta di messaggio per i posteri.

Prima che le spoglie mortali del dignitario e diplomatico polacco riposassero sotto l'altare della natio polona a Padova, il suo legame con i polacchi che giungevano nella città situata sulle rive del Bacchiglione era stato attestato da Hieronim Ręczajski². Questi, consiliarius dell'associazione patavina degli studenti polacchi, eletto a tale carica nel 1595, annotò nel libro della natio di aver depositato nella cassa "tre monete ungheresi” a nome di Stanisław Miński (Barycz, 1971, pp. 32, 332; Lenart, 2018, pp. 14-73). Questo dono, destinato all'erezione dell'altare, cosa perorata dagli studenti, fin dalla nascita della loro organizzazione, fondata nel 1592, saldamente legata alle riforme della Chiesa che manteneva i suoi legami con Roma, si dimostrò con il passare degli anni non solo una testimonianza della devozione del donatore, bensì in primo luogo un gesto di comprensione nei confronti della condizione umana, il che trovò espressione nell'iscrizione sopraindicata, incisa sulla lapide e collocata presso l'altare polacco. Józef Korzeniowski, dando alle stampe alla fine del XIX secolo il testo intitolato Sposób odprawowania poselstwa z obedyencyja do Rzymu (Maniera di fare ambasceria in obbedienza a Roma), si interessò più da vicino del luogo di eterno riposo di Miński, conosciuto, se non altro, grazie alle sue trascrizioni, già nu-

2 Hieronim Ręczajski, in seguito, arcidiacono di Cracovia, custode di Kielce e cancelliere del Cardinale Bernard Maciejowski. 
merose all'epoca, della dicitura presente sulla lapide (Lenart, 2019b, p. 32). Ne reca testimonianza l'editore, allegando l'informazione nella nota destinata ai lettori della trascrizione da lui preparata:

Per cortese intercessione del prof. Jan Marinelli ricevetti a Padova la suddetta scritta [si tratta del testo dell'iscrizione, precisazione mia ML], nonché la supplica di Erazm Dembiński dall'eminente conoscitore della storia di Padova, prof. Andrea Gloria, direttore del Museo Civico. A entrambi porgo i dovuti ringraziamenti. (Korzeniowski, 1889, p. 7)

Per saperne di più sulla sepoltura Korzeniowski si avvalse dunque dei suoi contatti con l'eminente geografo Giovanni Marinelli, che all'epoca lavorava presso l'Università di Padova, il quale a sua volta lo indirizzò da uno studioso che si occupava della storia dell'accademia di Padova. Si trattava dello stimato paleografo Andrea Gloria, editore delle fonti concernenti i primi secoli di vita dell'ateneo, dalla sua fondazione nel 1222, fino alla caduta di Padova sotto la dominazione veneziana, avvenuta nel 1405 (Barycz, 1927, p. 316). Gloria inviò a Korzeniowski, editore dell'opera di Miński, la trascrizione, conservata in archivio, della supplica di Erazm Dembowski, decano di Cracovia, parroco di Wieliczka e segretario reale, in privato cognato del defunto. Quello stesso membro della famiglia del sottocancelliere della Corona del Regno di Polonia, che si trovava a Padova allorché sulla strada del ritorno da Napoli, il giorno 21 luglio, ebbe fine il cammino terreno del diplomatico polacco, si rivolse per lettera al Consiglio Comunale di Padova in merito all'erezione del monumento. Dopo aver ottenuto il nullaosta richiesto, egli fece incidere sulla lapide il seguente testo, senza mancare di immortalare all'occasione anche il proprio cognome:

STANISLAVS MINSKI | DE MINSKO PALLATINVS | LANCICIEN: CANCELLARIVS | REGNI POLONIAE | MENS REGIS ET REGNI | VLTIMVM DECVS ET SPES | FAMILIAE | OBIIT PATAVY ANNO MDCVII | JVLY XXI AETATIS XLIV. | ERASMUS DEBINSKI DECANVS | CRAC. AFFINIS ET EXECR TESTI F.F. | O VITA FALLAX QVAE DESINIS | DVM VIVERE EXPEDIT. (Gonzati, 1852, pp. 240-241) 
Stanisław Miński di Mińsk, voivoda di Łęczyca, cancelliere del Regno di Polonia, persona di fiducia del Re, massimo decoro del regno e speranza della famiglia, morì a Padova nell'anno 1607, il 21 luglio, nel suo 44 anno di vita. Erazm Dembiński, decano di Cracovia, cognato ed esecutore testamentario fece erigere. Oh vita fallace, che desisti quando vivere occorre!

Merita di essere sottolineato il fatto che Dembowski, con la succitata supplica, abbia chiesto il permesso di poter erigere il monumento a Stanisław Miński non ai padri francescani, bensì al Consiglio Comunale di Padova. Lo facciamo notare poiché ciò significa che era perfettamente al corrente del carattere specifico del santuario, sottoposto alla giurisdizione della municipalità in quanto sua proprietà, cosa che spesso sfuggiva ai polacchi che soggiornavano a Padova. La Basilica di Sant'Antonio fu infatti amministrata per diversi secoli da un'istituzione appositamente ad essa preposta, la Veneranda Arca di Sant'Antonio, il cui atto ufficiale di fondazione risale al 1396 (Lenart, 2019d, pp. 57-64). Il testo di tale supplica, nella traduzione italiana, è il seguente:

Humanamente videndo, gloriosamente | morì la già felice memoria dell' $\mid$ Illustrissimo Signor Stanislao Min-| schi Palatino di Lancitia Cancel-| liero della Maestà del Re di Polo-| nia, il corpo del quale imbalsamato | fu servato nella Chiesa del Glorio-| \{so | Sant'\}|| Sant'Antonio, sin tanto s'havessero | nove dall' 'Illustrissima sua Madre | Palatina di Cracovia, quello del cadavero | far si dovesse, intentione | sua è stata, che si sepelischi qui | in Padova Terra di Studio, nella | qual concorrono ogni sorte di Na-| tioni con abito corrispondente | alla grandezza del titolo, et all'alto | suo Lignaio, questa cura è stata in-| gionta a me Erasmo Dembiski de | Velliani Vicario di Cracovia, et | Cognato del già deffonto Signore, | che per esser devotissimo di ques-| to Gloriosissimo Santo mio Inter-| cessore, et uno de quatro Prottet-| tori di questa Magnifica Città, | ho tra me stesso pensato, di darli || loco in questa sua Chiesa predicata | tra noi Polachi per la più famosa, |e ben composta, che si trovi in tutte | le parti del Mondo, alla qual, et per | orar, et per vederla concorrono d'ogni | intorno Signori Principi, Duchi, Re, | et Imperatori, ma perché far non | si può senza licenza delle Vostre Si-| gnorie Molt'Illustri, come quelle | quali sta il conceder tal gratia. Pe-| rò io Erasmo sudetto riccorro alle | loro benignità pregandole et suppli-| candole a volersi degnar de farci | gratia de loco in detta Chiesa | per mezo l'altare della nostra | Nation Polona, per fare una sepol-| tura a terra in modo tale, che non | eccedi il pavimento, né levi 
il | \{transito $\}||$ transito alle genti, che per ivi pas-| serano, simile a quelle, che sono sot-| to confessione nella sua Chiesa | catedrale dell'Illustrissimo Cardi-| nale Cornaro, Assicurandole che la | pietra, che per sepoltura servi-| rà, eccederà di valor, et di bellezza | le pietre, che si leverano, quali po-| trano servire per altre opere $\mid$ che in detta Chiesa far si potes-| sero. Gratia che se a me, che hu-| milmente gliela chiedo, mi sarà $\mid$ concessa, et di quella ne sarò fatto $\mid$ degno, oltre che tutta la nostra $\mathrm{Na}-\mid$ tione ne riceverà favor singo-| larissimo, io in particolare, et l'Illus-| trissima sua Madre ne teniremo | singolarissima memoria, et l'una || et l'altra delle nostre famiglie res-| terano eternamente obligatissime | alle Vostre Signorie Molt'Illustri, | alle quali di vivo core mi raccoman-| do. |

Io Girolamo Zacco Deputa<to $>$ veduto il loco | giudico il Molt'Illustre supplicante | esser degno di gratia, con questa con-| ditione però, che sia fatto il coperchio | della sepoltura sia de pietra de pa-| ragon, et il frizo sia di pietra corris-| pondente a questa. |

Qua supplicatione lecta in eodem | Magnifico Consilio; et posita | iudicio ultrascripti Magnifici | Consilii, datis, collectisque suffragijs | inventa fuere contraria decem, | et favorabilia octuaginta quatuor, | et capta, et concessa. || (Wrana, 2019, pp. 259-260)

Nel testo qui riportato vale la pena di evidenziare alcune informazioni per noi importanti. Prima di tutto la commemorazione di Miński presso l'altare ebbe luogo con la posa di una lastra di pietra nella pavimentazione, il che per secoli attirò l'attenzione dei pellegrini. Nel diario di viaggio stilato probabilmente dal viaggiatore lituano Michał Butler, alla data del 7 maggio 1780 troviamo la seguente breve annotazione: "Presso i padri francescani vi è a sinistra vicino alla porta l'altare di san Stanislao, dove riposano le sue reliquie, cioè sono esposte; nei pressi dell'altare è eretta la tomba dei polacchi, in cui si seppelliscono quelli deceduti per caso a Padova, come mausolei vengono mostrati quelli di Sapieha Aleksander Kazimierz (grande principe lituano) depiferis e Stanisław Miński de Minsko, voivoda e cancelliere del grande principe lituano sotto re Sigismondo" (Chorążyczewski, Pacevičius, \& Rosa, 2013, p. 323; Tygielski \& Kalinowska, 2019, p. 355).

Ricordiamo altresì che Stanisław Staszic nel suo Dziennik podróży (Diario di viaggio) del 1789-1805 annotò: "In quella stessa chiesa di Sant'Antonio vidi la tomba di alcuni polacchi: Ossoliński, Ponętowski, Miński, i quali giovani nelle scuole di là morirono" (Leśniewski, 1931, 
pp. 47-48; Lenart, 2005, p. 39). Alla soglia del XIX secolo, questa iscrizione non sfuggì all'attenzione di un "certo polacco", recatosi in Italia negli anni 1815 e 1816. Egli scrisse, dopo aver fatto visita al sepolcro del Santo Taumaturgo:

La chiesa di sant'Antonio a Padova è nota più per la buona fede nei miracoli del suo Santo Patrono che non per la fama della sua architettura gotica. Già all'entrata trovai la tomba di Stanisław Miński, cancelliere, morto nel 1607. Giace ivi anche il giovane Mikołaj Ponętowski; il fratello Andrzej gli eresse questo monumento nel 1548. (Wyjątki z podroży do Włoch pewnego Polaka w latach 1815 i 1816 [Estratti del viaggio in Italia di un certo polacco negli anni 1815 e 1816], 1819, p. 228)

Dall'inizio del XIX secolo molte guide scritte della Basilica di Sant'Antonio aiutavano i visitatori a individuare la lapide commemorativa di Miński. Troviamo menzione della sepoltura del voivoda di Łęczyca, con tanto di iscrizione trascritta, ad esempio nello stampato di padre Angelo Bigoni OFMConv., monaco eccezionalmente attivo come scrittore, che per un certo tempo rivestì la carica di ministro generale dell'ordine (Bigoni, 1816, p. 69; Marangoni, 1860). Ovviamente, l'autore non trascurò neppure altri monumenti funebri e lapidi legati ai polacchi, sebbene, come accade solitamente nel caso di autori italiani, decifrare i cognomi fosse per lui impresa ardua. Una pietra miliare negli studi sulla Basilica di Sant'Antonio fu l'opera in due volumi dello storico padovano padre Bernardo Gonzati OFMConv. (Gonzati, 1852; Gonzati, 1853), menzionato all'inizio nel contesto dell'edizione da parte di Józef Korzeniowski dello Sposob odprawowania poselstwa (Maniera di fare ambascerie). Occorre rilevare che proprio a quello studio attinse l'editore per il testo inciso sulla lapide di Miński. Dell'opera trattata in questa sede si avvalse del resto anche Józef Ignacy Kraszewski, il quale scrisse le sue memorie alcuni anni dopo il suo viaggio in Italia. La testimonianza di quest'ultimo riveste un certo interesse anche dal punto di vista letterario, quindi vale la pena di riportarla. Il gruppo di polacchi con cui si ritrovò nel santuario, ovviamente si diresse subito alla tomba di Sant'Antonio, e solo all'uscita ebbe inizio l'avventura così descritta: 
$\mathrm{Ci}$ apprestavamo ad uscire quando presso l'altare, proprio in vicinanza della porta, camminando a testa bassa, posai per caso lo sguardo sulla parete e, con eccezionale commozione, come se sentissi una voce provenire dalla tomba, lessi queste poche parole in polacco:

Karolina, dei conti Woyna Jabłonowska

Passò a miglior vita in un paese straniero

Trovò riposo e patria sotto quest'altare

Tra le spoglie dei suoi connazionali.

Deceduta a Venezia XVII. Gennaio MDCCCXL. -

È difficile esprimere quale profonda impressione fecero su di me quelle parole inaspettatamente rinvenute... Osservavo, standomene come impalato, la lapide con il bassorilievo di Luigi Ferrari, eccellente scultore dei nostri tempi, che rappresenta una figura femminile, la quale stringe una croce al petto e l'angelo che le porta la corona della Vittoria. - Più in basso c'era anche la dicitura in italiano [...]. Da questa scritta seppi che si trattava di un sepolcro nazionale, sollevai lo sguardo e solo allora scorsi nella pala d'altare il Martirio di San Stanislao. Guardandomi interno più attentamente, sulle pareti e sul pavimento scorsi alcune lastre, iscrizioni e stemmi polacchi, o con nomi polacchi... L'altare era evidentemente stato donato dai polacchi, e sotto le sue ali stringeva i defunti in terra straniera... Più in alto, sopra alla tomba della principessa Jabłonowska anneriva la pietra di Aleksander Kazimierz Sapieha, morto qui nel 1599, il 30 novembre. Sull'impiantito giaceva la lapide di Stanisław Miński, voivoda di Łęczyca, morto nel 1607, il 21 luglio [sottolineatura mia ML), nella cui tomba era stata sepolta anche la principessa Jabłonowska e alcuni altri, a testimonianza dei loro compaesani qui deceduti. (Kraszewski, 1866, pp. 170-171)

Leggendo le memorie dei viaggiatori polacchi non si possano ignorare i libri probabilmente più letti, il cui autore fu don Wincenty Smoczyński, figura alquanto colorita e meritevole per la "questione polacca", e al contempo organizzatore di numerosi pellegrinaggi a Roma (Róg, 1999, pp. 211-214). Quanto a Padova, egli fece interessanti osservazioni sulla Basilica di Sant'Antonio nelle memorie del suo pellegrinaggio, compiuto nel 1888 (Smoczyński, 1889, pp. 143-158), dove incluse anche parecchie informazioni su Miński, che di sicuro venivano lette dai pellegrini nel corso dei viaggi, che a quel tempo si effettuavano già in treno, il che forniva ulteriori opportunità di approfondire le proprie conoscenze sui monumenti visitati e sui personaggi ad essi legati già nel corso del viaggio. Smoczyński scrisse fra l'altro: 
Il giorno 15 gennaio 1594 giunse a Roma l'ambasceria di Sigismondo III per fare atto di obbedienza a Clemente VII, eletto papa, in precedenza nunzio apostolico in Polonia. Alla testa di tale ambasceria c’era Stanisław Miński, sottocancelliere della Corona del Regno di Polonia e voivoda di Łęczyca. Di questa ambasceria possediamo menzione nell'orazione tenuta durante la messa funebre, celebrata nella chiesa di Santa Barbara, per l'anima di sua madre, Dorota di Ojźrzanów, in prime nozze Mińska, in seconde nozze Barzyna, nella quale così disse l'anonimo predicatore: "Decoro della nostra Rzeczpospolita, senatore e voivoda e sottocancelliere, a tutta la Corona del Regno di Polonia noto e benemerito. Celebre per la legazione che da Sua Eccellenza il Re al Santo Padre Clemente VII diresse e atto di obbedienza alla Santa Sede a nome di Sua Maestà il Re fece. Con quale decoro e con quale gloria della nostra Patria, chi questo non sa? Il regno spagnolo e quello francese a quel tempo gli occhi verso la Polonia rivolsero, meravigliandosi di quanto benessere vedessero, e in così gran numero (aveva fino a trecento bravi uomini) e un tempo tanto lungo (giacché sei mesi circa) e giorno per giorno viveva come un signore, e ciò che la Polonia più degli altri stati può, fece sapere con dovizia. E non solo tale effimera gloria fondata su cose effimere a sé e ai fratelli suoi procurò, ma anche riempì la Chiesa Romana di religione e della particolare devozione del nostro popolo, allorché a nome della patria tutta davanti al Vicario di Cristo per la canonizzazione di San Giacomo del 1594 si inchinò. E tanto felicemente in tale ardua questione operò, che ciò che prima onorevoli principi e i cardinali stessi ottenere non poterono, quello lo stendardo della gloria del nostro Patrono per primo raccolse ed egli stesso nella nostra casa introdusse”. (Smoczyński, 1889, pp. 372-373)

\section{Egli non lesinò lodi a Miński nemmeno nella nota alla sua argomen- tazione:}

Aveva anche l'incarico di adoperarsi affinché venisse annoverato fra i Santi il domenicano Jacek Odrowąż, che nel 1594 con magnifiche cerimonie, per cura e in parte a spese di Miński solennemente fu dichiarato. Miński non solo stupì gli italiani con il suo intelletto, ma anche seppe con la sua dolcezza piacer loro. Papa Clemente in una lettera al Re ammise che lo aveva come "onestissimo uomo stimato, come piacevolissimo amato". (Smoczyński, 1889 , pp. 373-374, nota 1 )

Nella stessa nota Smoczyński incluse anche informazioni sull'ultimo viaggio di Miński in Italia, sul suo decesso a Padova, sulle esequie, e menzionò persino l'edizione della sua opera sulla maniera di condur- 
re un'ambasceria da parte di Józef Korzeniowski (ibid.). Aggiungiamo che, a cavallo dei secoli XIX e XX, un altro letterato, Władysław Bełza, lasciò un'importante relazione (Bełza, 1910), in cui menziona la lapide funebre di Minski, rimossa a causa dell'ennesimo rifacimento della pavimentazione.

Tornando al testo della supplica di Erazm Dembowski riportato sopra, appare essenziale il fatto di aver sottolineato i motivi per cui la madre del defunto aveva preso la decisione di seppellire la salma a Padova. Il cognato di Miński riporta infatti circostanze che, a suo giudizio, dovevano certamente fare impressione sui destinatari del testo. Egli si richiama in primo luogo alla frequentazione della città bagnata dal Bacchiglione, celebre per l'università che vi opera ("terra di scienza") da parte di studenti che rappresentavano svariate nazioni. La decisione di inumare la salma nel santuario è connessa non solo alla particolare devozione a Sant'Antonio, nonché al fatto che esso sia particolarmente caro ai polacchi, ma anche alla bellezza che lo contraddistingue. Aggiunge altresì che proprio là convergono da tutto il mondo sovrani, dignitari e aristocrati, per pregare sulla tomba del Grande Taumaturgo e ammirare la bellezza di questo luogo. Aggiungiamo che Miński riposa sotto l'altare, sulla cui pala è stato collocato un dipinto di Pietro Malerba che mostra i due patroni della Polonia, San Stanislao e San Giacomo Odrowąż (Lenart, 2019b, p. 163). Come già ricordato, Miński aveva svolto un ruolo importante nel processo di canonizzazione del secondo. Dal documento pervenutoci veniamo a sapere che prima questo venne letto il 20 ottobre 1607, durante la seduta del Consiglio dei Sedici e che, in seguito, il 12 novembre dello stesso anno, il Consiglio Comunale rilasciò il nullaosta, con 84 voti a favore e 10 contrari. Tuttavia, fu posta la condizione che sia il coperchio della tomba sia il fregio attorno ad essa fossero realizzati in marmo (pietra di paragone) (ASP, SA 3; Wrana, 2019, pp. 214-215). Tutto ciò non esaurì gli sforzi intrapresi, visto che tre anni dopo, il 22 gennaio 1611, questa volta Krzysztof Kochanowski, cortigiano reale ed amico del defunto, si rivolse al capitolo del convento con la preghiera di poter erigere a quello "un monumento eterno d'amicizia" (perpetuo monumento dell'amicizia) (ASP, SA 1). In definitiva, fu realizzata una lapide funebre in marmo (pietra di paragone), con le let- 
tere dell'epitaffio fuse in bronzo, collocata sul pavimento presso l'altare eretto dagli studenti polacchi. Aggiungiamo ancora un'osservazione riguardante i materiali d'archivio di cui disponiamo. Un loro attento lettore si domanderà, per quale motivo Dembiński e Kochanowski rivolgano la loro richiesta a persone che rappresentano prima il potere laico e poi quello religioso. Ricordiamo la lettera di Kochanowski:
$\mathrm{M}<$ ol $>$ to $\mathrm{R}<$ everen $>$ di Padri,
La memoria pia dell'Ill $<$ ustrissi $>$ mo Stanislao Minskii vicecancel-| lario del regno di polonia passato in megliore vita tre anni $\mid$ sono, et sepelito in padova, me spinge a lasciargli un perpetuo $\mid$ monumento dell'amicitia, che fu tra noi, Però la mia intentioni sarebbe drizzarli un monumento nella $\mathrm{Mag}<$ nifi $>$ ca Chiesa | del Santo a lato dell'Altare della Natione Polona; come $\mid$ anche per ornamento di detta Chiesa; piacendo alle $\mathrm{S}<$ ignorie $>\mathrm{V}<$ ostre $>\mid \mathrm{M}<$ ol $>$ to $\mathrm{R}<$ everen $>$ de del che le supplico; confidandomi, che havendo ri-| guardo ad una dimanda così pia et iusta, non impediranno | il mio proposito. Et gli Baccio le mani. |
Padova à dì 22 Gennaro 1611 .
Delle $\mathrm{S}<$ ignorie $>\mathrm{V}<$ ostre $>\mathrm{R}<$ everendissim $>$ e devoto $\mid$
Christophoro Cochan[[...]]wschii ca-| merario secreto della maestà di | Po- lonia etc. || (Wrana, 2019, p. 260)

Questo documento dimostra in maniera inconfutabile che tre anni dopo la morte di Miński non si era ancora riusciti ad erigere sulla sua tomba il relativo monumento funebre, cosa di certo non ostacolata dalla mancanza di fondi. Tornando al quesito che ci siamo posti in precedenza, siamo in grado, sulla base della seconda lettera sopraccitata, di confermare il fatto, già menzionato, che l'amministrazione della Basilica di Sant'Antonio era affidata (e parzialmente è tuttora così) ad un'istituzione laica, chiamata Veneranda Arca di Sant'Antonio, che opera, fra l'altro, negli spazi occupati dai monaci. A loro, del resto, si rivolgevano di solito con le loro richieste i polacchi, ignari della specificità della divisione dei ruoli a riguardo dell'amministrazione della basilica. Leggendo la supplica rivolta da Kochanowski, possiamo inoltre affermare con ampio margine di probabilità, che alla base della reiterazione della richiesta e del suo inoltro ai rappresentanti del capitolo stavano le tensioni ed i conflitti venutisi a creare nei secoli fra il potere laico ed i fran- 
cescani del convento (Lenart, 2019d, pp. 57-64). Proprio questo deve essere stato il motivo principale del fatto che la lapide sia stata collocata solo nel 1611, quando i padri francescani non si sentivano più messi in disparte in relazione alle decisioni connesse all'utilizzo degli spazi interni della basilica, tanto più che l'esercizio del culto al suo interno era, in fondo, di loro quasi esclusiva competenza.

La testimonianza costituita dai documenti sopraindicati, che dimostrano la determinazione nel voler conseguire lo scopo prefissosi, permettono di guardare alla lapide posta ai piedi dell'altare polacco come ad un elemento essenziale della promozione della cultura polacca, in uno spazio in cui essa acquistava un ulteriore significato. Nel corso del secolo successivo essa si sarebbe iscritta nel contesto della presenza attiva dei polacchi a Padova, i quali manifestavano sovente la propria specificità culturale e religiosità, di cui sono prova in parte le tracce della loro presenza raccolte nel santuario. In tale contesto essa divenne un messaggio rivolto ai posteri e, malgrado la lapide stessa sia stata rimossa, l'efficacia di questo messaggio è avvertibile ancora oggi, come dimostra in qualche misura anche il presente testo.

\section{BIBLIOGRAFIA}

Barycz, H. (Ed.). (1971). Archiwum nacji polskiej w Uniwersytecie Padewskim, t. 1: Metryka nacji polskiej w Uniwersytecie Padewskim 1592-1745 (index: K. Targosz). Wrocław: Ossolineum.

Barycz, H. (Ed.). (1972). Archiwum nacji polskiej w Uniwersytecie Padewskim,

t. 2: Statuta oraz Akta i protokoły nacji polskiej w Uniwersytecie Padewskim. Wrocław: Ossolineum.

Barycz, H. (1927). Zarys historiografii Uniwersytetu Padewskiego. Minerwa Polska, I/4, 311-323.

Bełza, W. (1910). Z Wenecyi do Neapolu: wrażenia z podroży. Kraków: S.A. Krzyżanowski; Warszawa: E. Wendego (T. Hiż i A. Turkuł).

Bigoni, A. (1816). Il forestiere istruito delle meraviglie e delle cose piu belle che si ammirano internamente ed esternamente nella basilica del gran taumaturgo s. Antonio di Padova con una raccolta intera e ordinata di tutte le iscrizioni della chiesa e de' chiostri e con l'aggiunta di tutte le 
sacre funzioni che vi si eseguiscono fra l'anno [...]. Padova: Stamp. del Seminario.

Chorążyczewski, W., Pacevičius, A., \& Rosa,A.(Eds.). (2013). Butleriokelionès i ItalijąirVokietija 1779-1780 metaisdienoraštis / Dziennik podróży Butlera do Włoch i Niemiec w latach 1779-1780. Vilnius: Vilniausuniversitetas / Vilniausuniversitetoleidykla.

Del Negro, P., \& Piovan, F. (Eds.). (2017). L'Università di Padova nei secoli (1222-1600). Documenti di storia dell'Ateneo. Treviso: Antilia.

Gonzati, B. (1852). La Basilica di S. Antonio di Padova descritta ed illustrata, t. 1. Padova: Antonio Bianchi.

Gonzati, B. (1853). La Basilica di S. Antonio di Padova descritta ed illustrata, t. 2. Padova: Antonio Bianchi.

Gruszecki, S. (1976). Miński Stanisław (dictionary entry). In Polski Stownik Biograficzny, t. 21 (pp. 321-322). Wrocław-Warszawa-KrakówGdańsk: Ossolineum.

Korzeniowski, J. (Ed.). (1889). Stanisława Mińskiego (1563-1607) sposób odprawowania poselstwa z obedyjencyją do Rzymu. Kraków: Akademia Umiejętności.

Kowalczyk, J. (1967). La Cappella della "Nazione Polacca" a Padova nel Seicento, Il Santo. Rivista antoniana di storia, dottrina, arte, VII/1, 67-86.

Kraszewski, J. I. (1866). Kartki z podroży 1858-1864 r., księga 1: Kraków, Wiedeń, Triest, Wenecja, Padwa, Medjolan, Genua, Piza, Florencja, Rzym. Warszawa: Gustaw Sennewald.

Lenart, M. (2010). Epitaphium Cretcovii świadectwem kontaktów padewskich Jana Kochanowskiego z otoczeniem Alvisa Cornara? In G. Urban-Godziek (Ed.), Twórczość Jana Kochanowskiego w kontekście nowołacińskiej literatury europejskiej i polskiej (pp. 64-75). Kraków. Retrieved from http://neolatina.bj.uj.edu.pl/neolatina/main/reports.html

Lenart, M. (2013). Patavium, Pava, Padwa. Tło kulturowe pobytu Jana Kochanowskiego na terytorium Republiki Weneckiej. Warszawa: Instytut Badań Literackich PAN.

Lenart, M. (2018). Album Polonicum. Metryka nacji polskiej w Padwie 15921745. Wstęp do edycji fototypicznej. In Album Polonicum. Metryka nacji polskiej w Padwie 1592-1745. Edycja fototypiczna (pp. 14-73). Warszawa: Narodowy Instytut Polskiego Dziedzictwa Kulturowego za Granicą POLONIKA.

Lenart, M. (2019a). Dzieje i znaczenie tzw. metryki nacji polskiej w Padwie: 1592-1745. Echa Przeszłości, XX/1, 143-164. 
Lenart, M. (2019b). Ołtarz nacji polskiej (1607-1809). In M. Lenart, M. Wojtkowska-Maksymik, \& M. Wrana, Polacy przy grobie św. Antoniego w Padwie, część 1: Pamiątki, donacje, świadectwa kultu (wieki $X V I-X I X)$ (pp. 145-187). Opole: Wydawnictwo Uniwersytetu Opolskiego.

Lenart, M. (2019c). Sobieski, la mazza di comando e i topi padovani. In G. Baldissin Molli (Ed.), La mazza e la mezzaluna. Turchi, Tartari e Mori al Santo (pp. 65-74). Padova: Edizioni Messaggero.

Lenart, M. (2019d). Świadectwa żywej obecności. Pamięć o Polsce i Polakach przy grobie św. Antoniego. In M. Lenart, M. Wojtkowska-Maksymik, \& M. Wrana, Polacy przy grobie św. Antoniego w Padwie, część 1: Pamiatki, donacje, świadectwa kultu (wieki XVI-XIX) (pp. 15-78). Opole: Wydawnictwo Uniwersytetu Opolskiego.

Lenart, M. (2005). Polscy podróżnicy w bazylice św. Justyny (XVI-XX w.). Opole: Wydawnictwo Uniwersytetu Opolskiego.

Leśniewski, C. (1931). Dziennik podroży Stanisława Staszica 1789-1805. Kraków: Polska Akademia Umiejętności.

Marangoni, L. (1860). Cenni storici sulla vita del reverendissimo padre maestro Angelo Bigoni, ex ministro generale dei Minori Conventuali [...]. Padova: A. Bianchi.

Róg, R. (1999). Smoczyński Wincenty (dictionary entry). In H. Markiewicz (Ed.), Polski słownik biograficzny, t. 39 (pp. 211-214). Wrocław-Warszawa-Kraków-Gdańsk: Ossolineum.

Luisetto, G. (Ed.). (1983). Archivio Sartori documenti di storia e arte francescana, t. 1: Basilica e convento del Santo. Padova: Biblioteca Antoniana - Basilica del Santo.

Smoczyński, W. (1880). Rzym, jego kościoły i pomniki: upominek pielgrzymom polskim. Kraków: Druk. "Czasu".

Smoczyński, W. (1889). Wspomnienia o polskiej pielgrzymce do Rzymu w roku 1888 na jubileusz j. ś. Leona XIII papieża. Kraków: s.n.

Tygielski, W., \& Kalinowska, A. (Ed.). (2019). W podróży po Europie. Studia $z$ dziejów kultury nowożytnej. Warszawa: Wydawnictwa Uniwersytetu Warszawskiego.

Szczurowski, R. (1999). Ksiądz Wincenty Smoczyński - przewodnik pielgrzymów do Rzymu w latach 1888-1900. Nasza Przeszłość, 91, 301-330.

Wrana, M. (2019). Mauzoleum nacji polskiej. Pochówki, nagrobki, tablice epitafijne (1558-1840). In M. Lenart, M. Wojtkowska-Maksymik, \& M. Wrana, Polacy przy grobie św. Antoniego w Padwie, część 1: 
Pamiatki, donacje, świadectwa kultu (wieki XVI-XIX) (pp. 192-203, 238-254). Opole: Wydawnictwo Uniwersytetu Opolskiego.

Wyjątki z podroży do Wloch pewnego Polaka w latach 1815 i 1816. (1819). Pamiętnik Lwowski, marzec, 212-235.

\section{MANOSCRITTI}

ASP, SA 1 (Padova, Archivio di Stato, Corporazioni Soppresse, Convento S. Antonio), b. 176, Veneranda Arca contro Città di Padova. Suppliche ai Padri del Santo per ottenere luoghi in chiesa e convento per erigere epitafi, memorie e cappelle, 1405-1683, fasc. N, c. 35r, 22.01.1611.

ASP, SA 2 (Padova, Archivio di Stato, Corporazioni Soppresse, Convento S. Antonio), b. 176, c. 19r-20r.

ASP, SA 3 (Padova, Archivio di Stato, Corporazioni Soppresse, Convento S. Antonio), b. 178, Padri Presidenti Regolari dell'Arca contro Magnifica Città e contro Presidenti Secolari. Tomo S, T, V, X, Z, 1405-1691, t. S, c. $65 \mathrm{v}-68 \mathrm{r}, 12.11 .1607$.

Riassunto: L'articolo affronta la questione della prima sepoltura nella cripta funebre, realizzata sotto l'altare della natio polona nella Basilica di Sant'Antonio a Padova. Tale sepoltura riguardava Stanisław Miński, dignitario di stato polacco e diplomatico, che passò da Padova diretto a Napoli. Dell'inumazione del corpo di Miński nel luogo di celebrazione liturgica del santuario associato alla Polonia ed ai polacchi informava una lapide, rimossa solo alla metà del XX secolo. Per oltre tre secoli essa attirò l'attenzione soprattutto dei pellegrini polacchi giunti a Padova, ricordando non solo la figura del grande diplomatico polacco, ma anche la natio polona, che nel secolo XVI e agli inizi del XVII costituiva in città un gruppo numeroso e particolare di oltremontani, in gran parte legati agli studi compiuti nel periodo d'oro dell'università. Nell'articolo sono riportati documenti scarsamente noti concernenti la sepoltura, conservati negli archivi padovani, redatti dall'amministrazione religiosa e da quella della basilica. Grazie alla loro analisi, è stato ricostruito il contesto culturale nel quale fu deciso di rinunciare al trasporto della salma di Miński in Polonia. Si è altresì sottolineato il ruolo svolto dall'istituzione cerata dall'amministrazione della Basilica di Sant'Antonio, operante con il nome di Veneranda Arca di Sant'Antonio, la quale rilasciava i permessi per l'inumazione delle salme nella basilica.

Parole chiave: Stanisław Miński, Padova, natio polona, Basilica di Sant’Antonio, tomba

\section{Traduzione dal polacco di Maurizio Mazzini}

